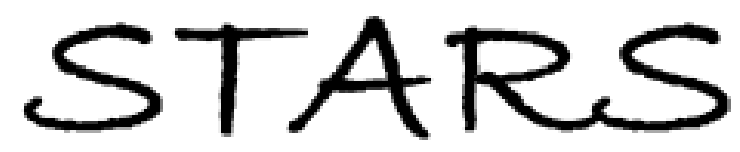

University of Central Florida

STARS

$1-1-2014$

\title{
Computed tomography image using sub-terahertz waves generated from a high-T-c superconducting intrinsic Josephson junction oscillator
}

\author{
T. Kashiwagi \\ K. Nakade \\ Y. Saiwai \\ H. Minami \\ T. Kitamura
}

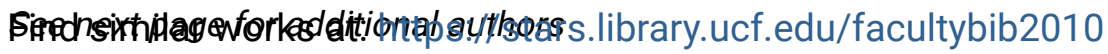

University of Central Florida Libraries http://library.ucf.edu

This Article is brought to you for free and open access by the Faculty Bibliography at STARS. It has been accepted for inclusion in Faculty Bibliography 2010 s by an authorized administrator of STARS. For more information, please contactSTARS@ucf.edu.

\section{Recommended Citation}

Kashiwagi, T.; Nakade, K.; Saiwai, Y.; Minami, H.; Kitamura, T.; Watanabe, C.; Ishida, K.; Sekimoto, S.; Asanuma, K.; Yasui, T.; Shibano, Y.; Tsujimoto, M.; Yamamoto, T.; Marković, B.; Mirković, J.; Klemm, R. A.; and Kadowaki, K., "Computed tomography image using sub-terahertz waves generated from a high-T-c superconducting intrinsic Josephson junction oscillator" (2014). Faculty Bibliography 2010s. 5556. https://stars.library.ucf.edu/facultybib2010/5556

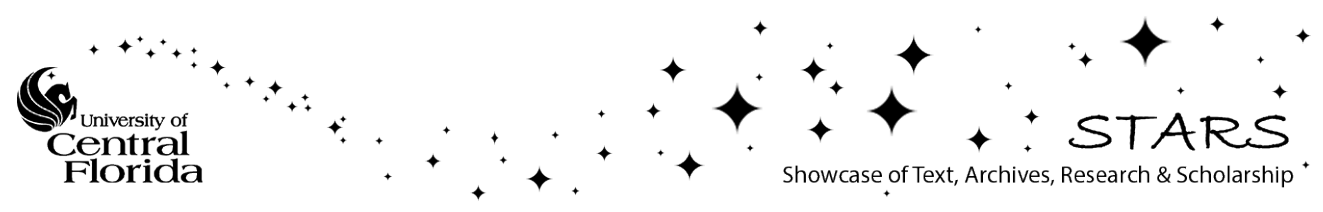




\section{Authors}

T. Kashiwagi, K. Nakade, Y. Saiwai, H. Minami, T. Kitamura, C. Watanabe, K. Ishida, S. Sekimoto, K. Asanuma, T. Yasui, Y. Shibano, M. Tsujimoto, T. Yamamoto, B. Marković, J. Mirković, R. A. Klemm, and K. Kadowaki 


\section{Computed tomography image using sub- terahertz waves generated from a high- $T_{C}$ superconducting intrinsic Josephson junction oscillator}

Cite as: Appl. Phys. Lett. 104, 082603 (2014); https://doi.org/10.1063/1.4866898

Submitted: 04 December 2013 . Accepted: 29 January 2014 . Published Online: 28 February 2014

T. Kashiwagi, K. Nakade, Y. Saiwai, H. Minami, T. Kitamura, C. Watanabe, K. Ishida, S. Sekimoto, K. Asanuma, T. Yasui, Y. Shibano, M. Tsujimoto, T. Yamamoto, B. Marković, J. Mirković, R. A. Klemm, and K. Kadowaki

\section{ARTICLES YOU MAY BE INTERESTED IN}

Reflection type of terahertz imaging system using a high- $\mathrm{T}_{\mathrm{C}}$ superconducting oscillator Applied Physics Letters 104, 022601 (2014); https://doi.org/10.1063/1.4861602

A high- $T_{C}$ intrinsic Josephson junction emitter tunable from 0.5 to 2.4 terahertz Applied Physics Letters 107, 082601 (2015); https://doi.org/10.1063/1.4929715

Continuous $30 \mu \mathrm{W}$ terahertz source by a high-T superconductor mesa structure Applied Physics Letters 103, 182601 (2013); https://doi.org/10.1063/1.4827094

\section{Applied Physics Reviews} Now accepting original research 


\title{
Computed tomography image using sub-terahertz waves generated from a high- $T_{c}$ superconducting intrinsic Josephson junction oscillator
}

\author{
T. Kashiwagi, ${ }^{1,2, a)}$ K. Nakade, ${ }^{1}$ Y. Saiwai, ${ }^{1}$ H. Minami, ${ }^{1,2}$ T. Kitamura,,${ }^{1}$ C. Watanabe,${ }^{1}$ \\ K. Ishida, ${ }^{1}$ S. Sekimoto, ${ }^{1}$ K. Asanuma,${ }^{1}$ T. Yasui, ${ }^{1}$ Y. Shibano, ${ }^{1}$ M. Tsujimoto, ${ }^{3}$ \\ T. Yamamoto, ${ }^{4}$ B. Marković, ${ }^{5}$ J. Mirković, ${ }^{6}$ R. A. Klemm, ${ }^{7}$ and K. Kadowaki ${ }^{1,2}$ \\ ${ }^{1}$ Graduate School of Pure and Applied Sciences, University of Tsukuba, 1-1-1 Tennodai, Tsukuba, Japan \\ ${ }^{2}$ Division of Materials Science, Faculty of Pure and Applied Sciences, University of Tsukuba, 1-1-1, Tennodai, \\ Tsukuba, Ibaraki 305-8573, Japan \\ ${ }^{3}$ Department of Electronic Science and Engineering, Kyoto University, Nishikyo-ku, Kyoto 615-8510, Japan \\ ${ }^{4}$ Wide Bandgap Materials Group, Optical and Electronic Materials Unit, Environment and Energy Materials \\ Division, National Institute for Materials Science, 1-1 Namiki, Tsukuba, Ibaraki 305-0044, Japan \\ ${ }^{5}$ Faculty of Sciences, University of Montenegro, George Washington Str., 81000 Podgorica, Montenegro \\ ${ }^{6}$ Faculty of Science, University of Montenegro, and CETI, Put Radomira Ivanovica, 81000 Podgorica, \\ Montenegro \\ ${ }^{7}$ Department of Physics, University of Central Florida, 4000 Central Florida Blvd., Orlando, \\ Florida 32816-2385, USA
}

(Received 4 December 2013; accepted 29 January 2014; published online 28 February 2014)

A computed tomography (CT) imaging system using monochromatic sub-terahertz coherent electromagnetic waves generated from a device constructed from the intrinsic Josephson junctions in a single crystalline mesa structure of the high- $T_{\mathrm{c}}$ superconductor $\mathrm{Bi}_{2} \mathrm{Sr}_{2} \mathrm{CaCu}_{2} \mathrm{O}_{8+\delta}$ was developed and tested on three samples: Standing metallic rods supported by styrofoam, a dried plant (heart pea) containing seeds, and a plastic doll inside an egg shell. The images obtained strongly suggest that this CT imaging system may be useful for a variety of practical applications. (c) 2014 AIP Publishing LLC. [http://dx.doi.org/10.1063/1.4866898]

Recently, compact and powerful generators of electromagnetic waves at terahertz $(\mathrm{THz})$ frequencies based on semiconducting devices such as resonant tunneling diodes (RTDs), unitraveling carrier photo-diodes (UTC-PDs), quantum cascade lasers (QCLs), femtosecond current switching (CS) devices, etc., have been developed very rapidly, because such a frequency domain has became known to be highly useful not only for basic research in science and technology but also for a wide variety of many practical applications, with special emphasis on various kinds of imaging, high-speed communications, security inspections, medical diagnoses, pharmaceutical studies and development, biosciences and technologies, environmental studies, quality control of industrial products, quantum computing, etc. ${ }^{1,2}$

A unique $\mathrm{THz}$ source which generates monochromatic coherent continuous radiation was discovered in 2007 using high-transition temperature $T_{c}$ superconducting $\mathrm{Bi}_{2} \mathrm{Sr}_{2} \mathrm{CaCu}_{2} \mathrm{O}_{8+\delta}$ (Bi2212) mesa structures. ${ }^{3,4}$ Many characteristics of the $\mathrm{THz}$ devices based on Bi2212 sources have been studied experimentally and theoretically. ${ }^{5-22}$ The radiation characteristics from this source are entirely different from those of conventional semiconducting and optical sources because of the completely different radiation principle based upon the ac-Josephson effect, whereby the emission frequency $f=f_{\mathrm{J}}=K_{\mathrm{J}} V$, where $V$ is the dc voltage applied across each intrinsic Josephson junction (IJJ) (see below), and $K_{\mathrm{J}}=2 e / h=483.597891(12)[\mathrm{GHz} / \mathrm{mV}]$, where $e$ is the electronic charge and $h$ is Planck's constant. ${ }^{23}$ Since $f_{\mathrm{J}} \propto V$, it can be varied with $V$ rather arbitrarily. Furthermore, since the superconducting energy gap $2 \Delta$

${ }^{a}$ kashiwagi@ims.tsukuba.ac.jp $\approx 60 \mathrm{meV} \approx 28.8 \mathrm{THz}$ for Bi2212, these devices can in principle become excellent radiation sources even for $f$ ranging from $\sim 0.3 \mathrm{THz}$ to $\sim 20 \mathrm{THz}$. These novel sources have therefore accelerated the race to develop powerful, compact, and easy-to-use $\mathrm{THz}$ sources.

Bi2212, a highly anisotropic layered superconductor with $T_{c} \sim 90 \mathrm{~K}$, consists of alternating and mutually incommensurate double $\mathrm{CuO}_{2}$ layers responsible for its superconductivity and an insulating $\mathrm{Bi}_{2} \mathrm{O}_{2}$ block layer within each half crystallographic unit cell $c$-axis edge of $\sim 15.3 \AA$. Therefore, a Bi2212 single crystal $1 \mu \mathrm{m}$ thick corresponds to a $c$-axis stack of $\sim 653$ equivalent atomic-scale IJJs. ${ }^{24-26}$

Recently, we obtained emission with the output power of $\sim 30 \mu \mathrm{W}$ from a stand-alone mesa structure ${ }^{27}$ for $f$ between 0.3 and $1.0 \mathrm{THz} .{ }^{14,16,18}$ A synchronized three-mesa array was recently reported to emit with about $600 \mu \mathrm{W}$ output power. ${ }^{17}$ Such remarkable power development is comparable to those from other THz sources, such as RTDs, ${ }^{28-30}$ UTC-PDs, ${ }^{31,32}$ and QCLs. ${ }^{33-35}$ As for QCLs, the competition between the energy level separation and thermal energy forces the limitation $h f>k_{B} T$, for which $1 \mathrm{THz} \sim 48\left(k_{B} / h\right) \mathrm{K}$, where $k_{B}$ is Boltzmann's constant.

We developed transmission and reflection types of imaging systems for practical uses of our IJJ-THz emitter. ${ }^{13,36}$ The electromagnetic waves in the $\mathrm{THz}$ region can easily penetrate through papers and plastic materials, hence hidden objects with high absorption coefficients in the $\mathrm{THz}$ region can be detected by $\mathrm{THz}$ wave imaging systems. The transmission and reflection types of imaging systems may provide information regarding the bulk and surface structures of a sample, respectively. The different characteristics obtained from those two systems can enable us to identify highly reflective and 
highly absorbing materials within the lightly concealed sample. As discussed in the following, a THz-wave computed tomography (THz-CT) imaging technique can also provide cross-sectional views of the sample's internal structure. By combining these techniques efficiently, one can obtain unique images useful for practical applications based on the characteristic features of $\mathrm{THz}$ waves.

In this Letter, we describe a THz-CT imaging system using our IJJ-THz emitter as the source and present THz-CT images of three samples: Three standing metallic rods supported by styrofoam, a dried heart pea (cardiospermum helicacabum) with three seeds inside, and a plastic doll inside an egg shell.

Figure 1 shows a schematic top view of the THz-CT imaging system using our IJJ-THz emitter. The IJJ-THz emitter was placed in a He-flow cryostat (CF1104, OXFORD Instruments) and cooled down to the operating temperature between 10 and $70 \mathrm{~K}$. The $\mathrm{THz}$ electromagnetic waves generated from the IJJ-THz emitter are collected and converted to the parallel beam by the first mirror, $\mathrm{M}_{1}$, then it is focused to the sample by the second mirror, $\mathrm{M}_{2}$. The focal lengths of $M_{1}$ and $M_{2}$ are $152 \mathrm{~mm}$ and $220 \mathrm{~mm}$, respectively. The samples are placed on a rotating table mounted on a $2 \mathrm{D}(x, z)$ scanning stage (SGAHM26-200, SIGMA KOKI Co.). This scanner enables us to take one-dimensional images along the $x$ (horizontal) direction at various speeds below $130 \mathrm{~mm} / \mathrm{s}$ at various fixed rotation angles $\theta$ of the sample table in order to measure the angular and spatial dependencies of the transmission intensity $I(x, y, \theta)$ of the $\mathrm{THz}$ radiation. The transmitted $\mathrm{THz}$ beam from the sample is detected by a high-speed InSb hot-electron (HE) bolometer (QFI/2BI, QMC Instruments Ltd.), whose detection power was monitored by a lock-in amplifier (Model 7265, EG\&G instruments Co.). To facilitate the lock-in detection, the output power of the IJJ-THz emitter is effectively chopped by superposing an ac square-wave voltage of amplitude $80 \mathrm{mV}$ and frequency $10 \mathrm{kHz}$ onto the dc bias voltage with a function generator (33220A, Agilent). We note that lock-in detection using this electronic modulation is superior in noise performance to that of an optical chopper as commonly used for optical and infrared detection, because it

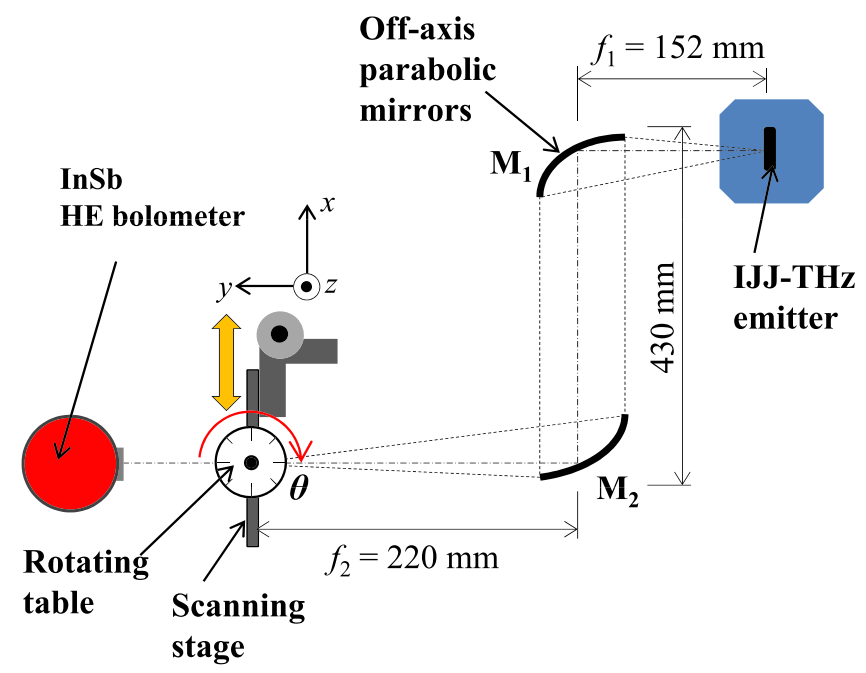

FIG. 1. A schematic top view of the THz-CT imaging system using an IJJTHz emitter. essentially eliminates the thermal background noise due to the surrounding environment from the detected signal. Further details of the detection method were described previously. ${ }^{13}$

In order to obtain reliable CT images, it is crucially important to have a $\mathrm{THz}$ source beam that is stable throughout the time of the measurements. For the present THz-CT imaging measurements, we selected an $\mathrm{IJJ}-\mathrm{THz}$ emitter with dimensions $80 \times 400 \times 2.4 \mu \mathrm{m}^{3}$, due to its stability and output power sufficient for the ac voltage bias modulation measurements. The ratio of the temporal variability $\Delta P(t)$ to that of the temporal average $\overline{P(t)}$ of the output power of the chosen IJJ-THz emitter during the time of each sample measurement was less than $\pm 1 \%$. This device was then operated at $45 \mathrm{~K}$ and emission $f=440 \mathrm{GHz}$. $f$ was easily estimated using our previous interference pattern technique. ${ }^{13}$

We note that our IJJ-THz emitter has a spatial distribution of its radiation power. Mirror $\mathrm{M}_{1}$ in Fig. 1 collects a part of this radiation power distribution. We adjusted the dc bias voltage to generate stable radiation, and the voltage of the lock-in detector of the bolometer response is about $V_{\text {det }}=40 \mathrm{mV}$ at this stable radiation bias point without any sample. According to our previous studies, ${ }^{18}$ this corresponds to the incident $\mathrm{THz}$ radiation power, $P=2 \sqrt{2} V_{\text {det }} / \alpha \approx 34 \mathrm{nW}$, where $\alpha=3.3 \mathrm{mV} / \mathrm{nW}$ is the system optical responsivity calibrated by black body radiation.

We note that the THz-CT images shown here are constructed by superposing the $\theta$ dependence of the 2D matrix $I(x, y, \theta)$ from each line scan along the $x$ direction based on the primitive filtered back projection CT imaging technique. ${ }^{37}$

We first made a simple check using a trivial test sample. Figure 2 shows the THz-CT imaging picture of the three standing metallic rods with the diameter of $2 \mathrm{~mm}$ supported by a styrofoam base. The inset of Fig. 2 shows an optical photograph of the sample. This THz-CT image was constructed by superposing the $\theta$-dependent images taken from $\theta=0$ to $360^{\circ}$ in $10^{\circ}$ steps, which were obtained by horizontal $(x)$ line scanning at the speed of $10 \mathrm{~mm} / \mathrm{s}$ in scanning steps

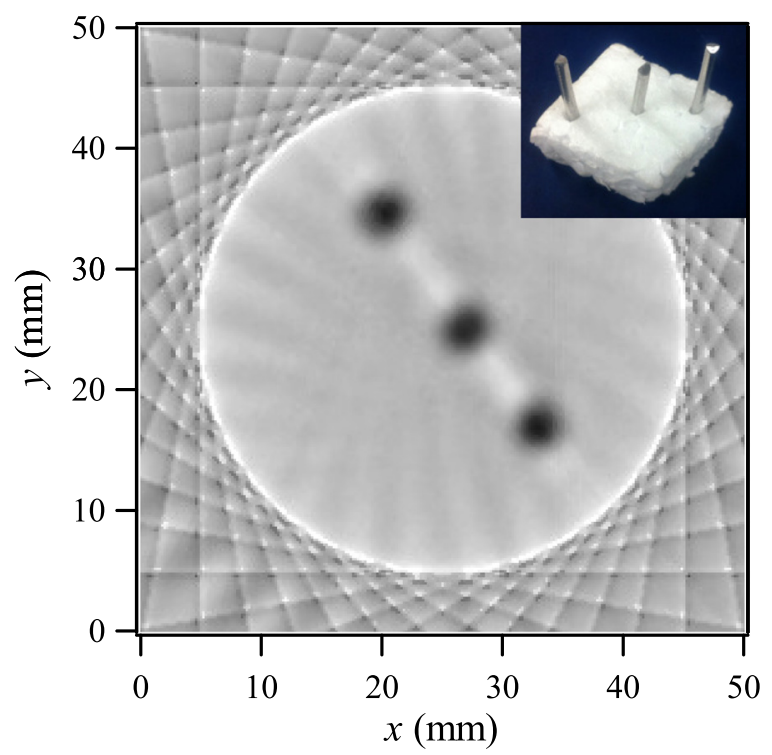

FIG. 2. A THz-CT image of three metallic rods each $2 \mathrm{~mm}$ in diameter supported by a styrofoam base. Inset: An optical photograph of the same supported metallic rods. 

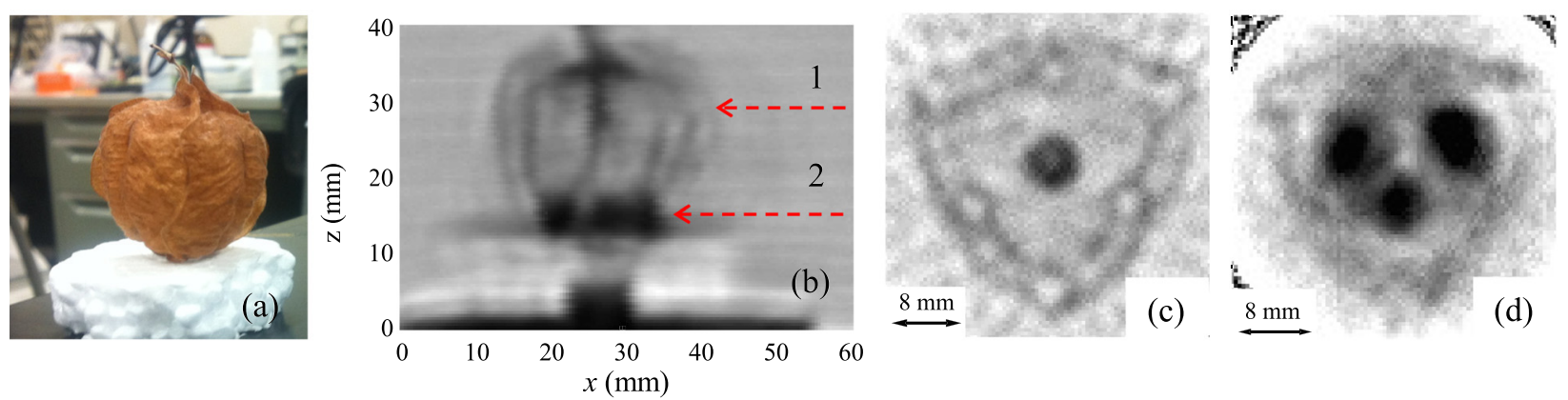

FIG. 3. (a) Optical photograph of the exterior of a dried heart pea. (b) Transmission image of the dried heart pea. (c) and (d) THz-CT horizontal planar images taken at fixed $z$ values indicated by dashed red arrows 1 and 2 in Fig. 3(b).

of $0.2 \mathrm{~mm}$. It took about $10 \mathrm{~min}$ to collect all angular dependent data. The resulting composite image shown in Fig. 2 clearly demonstrates the sample characteristics of the positions, the distances, and the diameters of three rods. From this image, we estimate the spatial resolution of our transmission imaging system to be on the order of or somewhat larger than $1 \mathrm{~mm}$. We note that the two upper left THz-CT rod images appear to have slightly larger diameters than that of the other rod, although they all have the same diameter. This may be due to the fact that the rods are not all standing parallel to the rotation axis. Moreover, the THz-CT detected diameters of all three rods are considerably bigger than $2 \mathrm{~mm}$. This is probably due to the resolution limit of our system, $\sim 1 \mathrm{~mm}$, obtained using monochromatic $\mathrm{THz}$ electromagnetic waves.

We now present the results of a THz-CT scan of a sample that is more relevant to the utility of the imaging system: A dried heart pea with three seeds inside. An optical photograph of the heart pea placed on a styrofoam base is shown in Fig. 3(a). The 2D transmission THz-image is displayed in Fig. 3(b). The three dark spots at the bottom of the heart pea are images of the three seeds located in three different inflated ventricles (or chambers) inside the heart pea that are separated by thin-shelled walls, an exterior view of which is seen in Fig. 3(a). The faint vertical lines connected at the top and bottom are the images of the three walls separating the ventricles of the heart pea. The THz-CT images of the heart pea constructed by 36 transmission $2 \mathrm{D}$ images at the horizontal planes indicated by the red dashed arrows 1 and 2 in Fig. 3(b) are shown in Figs. 3(c) and 3(d), respectively. Note that the horizontal images for the CT image are taken at a scanning speed of $10 \mathrm{~mm} / \mathrm{s}$ along $x$ in measurement steps of
$0.4 \mathrm{~mm}$. It is remarkable that Fig. 3(c) clearly shows the characteristic features of a heart pea: Three ventricles, each consisting of an inflated thin-walled, balloon-like triangular capsule, that are pressed together. In Fig. 3(d), three ball-like seeds located in separate ventricles are also clearly visualized at the bottom of the heart pea. These results provide strong evidence that the $\mathrm{THz}-\mathrm{CT}$ imaging technique is extremely useful to investigate the internal structures, distributions, textures, etc., of the object containing different $\mathrm{THz}$ absorption coefficients.

Next, we examine a sample consisting of a small plastic doll placed in an egg shell by the THz-CT technique. Figure 4(a) shows an optical photograph of the egg shell placed on and supported by the paper holder on the sample stage. A plastic doll was placed in the egg shell upside down as shown in Fig. 4(b). Then the egg shell is closed and the transmission image was taken. This result is shown in Fig. 4(c), where the head of the small doll, egg shell, and the paper holder can be seen rather vividly, although plastics in general have a very low absorption coefficient. It is clear that the $\mathrm{THz}$ waves easily penetrate through the egg shell, whereas the plastic doll has a slightly larger absorption coefficient than does the egg shell as seen in Fig. 4(c). The THzCT image obtained at the red dashed line in Fig. 4(c) is displayed in Fig. 4(d). This CT imaging picture was obtained with the line scanning speed of $10 \mathrm{~mm} / \mathrm{s}$ in steps of $0.2 \mathrm{~mm}$. We emphasize that the image in Fig. 4(d) clearly detects the characteristics of the body and hands of the doll, the position of the doll in the egg shell, and the edge of the egg shell. These remarkable results will accelerate further detailed studies of this $\mathrm{THz}-\mathrm{CT}$ imaging technique, especially for the variety of applications such as in bio- and life-sciences.
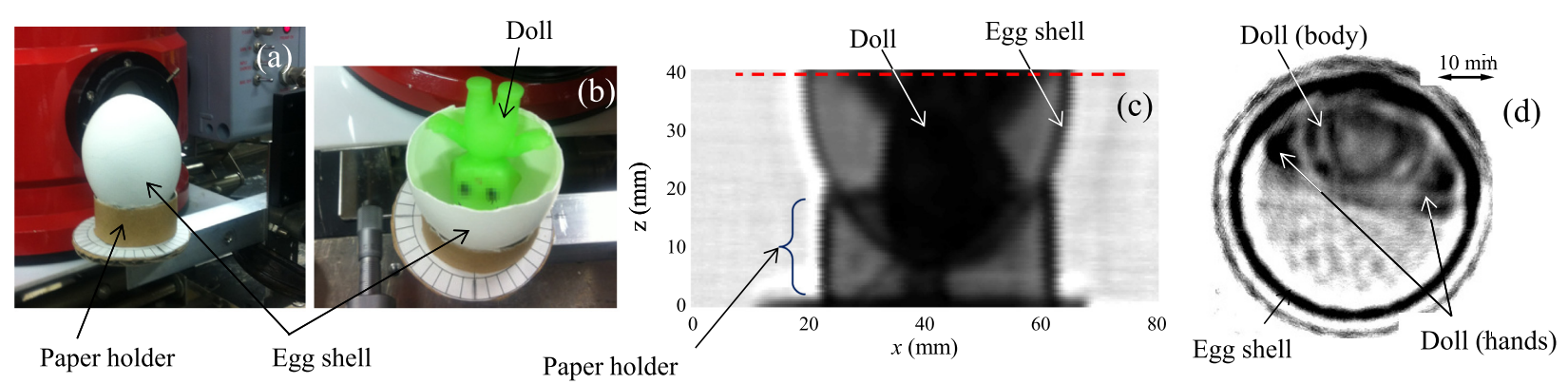

FIG. 4. (a) and (b) Optical photographs of an egg shell supported by a paper holder on the sample stage and of a doll placed upside down inside the egg shell. (c) Transmission image of the doll in the egg shell. (d) THz-CT image of the doll in the egg shell. 
In conclusion, we have shown three different $\mathrm{CT}$ images taken by the home-made THz-CT image scanning system at a sub-THz frequency $(440 \mathrm{GHz})$ generated from the mesa structure fabricated from single crystalline high- $T_{\mathrm{c}}$ superconducting $\mathrm{Bi}_{2} \mathrm{Sr}_{2} \mathrm{CaCu}_{2} \mathrm{O}_{8+\delta}$. We demonstrated with three different examples that our IJJ-THz emitter may be employed in transmission THz-CT imaging systems and, thereby, also vividly demonstrated the utility of the THz-CT scanning technique in future imaging applications.

This work was supported in part by the Grant-in-Aid for challenging Exploratory Research from the Ministry of Education, Culture, Sports, Science and Technology. T.K. thanks the Matsuda foundation and the JST A-STEP program for their financial support.

${ }^{1}$ B. Ferguson and X. C. Zhang, Nature Mater. 1, 26 (2002).

${ }^{2}$ M. Tonouchi, Nat. Photonics 1, 97 (2007).

${ }^{3}$ L. Ozyuzer, A. E. Koshelev, C. Kurter, N. Gopalsami, Q. Li, M. Tachiki, K. Kadowaki, T. Yamamoto, H. Minami, H. Yamaguchi, T. Tachiki, K. E. Gray, W.-K. Kwok, and U. Welp, Science 318, 1291 (2007).

${ }^{4}$ U. Welp, K. Kadowaki, and R. Kleiner, Nat. Photonics 7, 702 (2013).

${ }^{5}$ K. Kadowaki, M. Tsujimoto, K. Yamaki, T. Yamamoto, T. Kashiwagi, H. Minami, M. Tachiki, and R. A. Klemm, J. Phys. Soc. Jpn. 79, 023703 (2010).

${ }^{6}$ H. B. Wang, S. Guénon, B. Gross, J. Yuan, Z. G. Jiang, Y. Y. Zhong, M. Grünzweig, A. Iishi, P. H. Wu, T. Hatano, D. Koelle, and R. Kleiner, Phys. Rev. Lett. 105, 057002 (2010).

${ }^{7}$ T. M. Benseman, A. E. Koshelev, K. E. Gray, W.-K. Kwok, U. Welp, K. Kadowaki, M. Tachiki, and T. Yamamoto, Phys. Rev. B 84, 064523 (2011).

${ }^{8}$ M. Tsujimoto, T. Yamamoto, K. Delfanazari, R. Nakayama, T. Kitamura, M. Sawamura, T. Kashiwagi, H. Minami, M. Tachiki, K. Kadowaki, and R. A. Klemm, Phys. Rev. Lett. 108, 107006 (2012).

${ }^{9}$ B. Gross, S. Guenon, J. Yuan, M. Y. Li, J. Li, A. Ishii, R. G. Mints, T. Hatano, P. H. Wu, D. Koelle, H. B. Wang, and R. Kleiner, Phys. Rev. B 86, 094524 (2012).

${ }^{10}$ M. Li, J. Yuan, N. Kinev, J. Li, B. Gross, S. Guenon, A. Ishii, K. Hirata, T. Hatano, D. Koelle, R. Kleiner, V. P. Koshelets, H. Wang, and P. Wu, Phys. Rev. B 86, 060505 (2012).

${ }^{11}$ I. Kakeya, Y. Omukai, T. Yamamoto, K. Kadowaki, and M. Suzuki, Appl. Phys. Lett. 100, 242603 (2012).

${ }^{12}$ H. Minami, M. Tsujimoto, T. Kashiwagi, T. Yamamoto, and K. Kadowaki, IEICET Trans. E95-C, 347 (2012).

${ }^{13}$ M. Tsujimoto, H. Minami, K. Delfanazari, M. Sawamura, R. Nakayama, T. Kitamura, T. Yamamoto, T. Kashiwagi, T. Hattori, and K. Kadowaki, J. Appl. Phys. 111, 123111 (2012).
${ }^{14}$ T. Kashiwagi, M. Tsujimoto, T. Yamamoto, H. Minami, K. Yamaki, K. Delfanazari, K. Deguchi, N. Orita, T. Koike, R. Nakayama, T. Kitamura, M. Sawamura, S. Hagino, K. Ishida, K. Ivanovic, H. Asai, M. Tachiki, R. A. Klemm, and K. Kadowaki, Jpn. J. Appl. Phys., 51, 010113 (2012).

${ }^{15}$ F. Turkoglu, H. Koseoglu, Y. Demirhan, L. Ozyuzer, S. Preu, S. Malzer, Y. Simsek, P. Müller, T. Yamamoto, and K. Kadowaki, Supercond. Sci. Technol. 25, 125004 (2012).

${ }^{16}$ K. Kadowaki, M. Tsujimoto, K. Delfanazari, T. Kitamura, M. Sawamura, H. Asai, T. Yamamoto, K. Ishida, C. Watanabe, S. Sekimoto, K. Nakade, T. Yasui, K. Asanuma, T. Kashiwagi, H. Minami, M. Tachiki, T. Hattori, and R. A. Klemm, Physica C 491, 2 (2013).

${ }^{17}$ T. M. Benseman, K. E. Gray, A. E. Koshelev, W.-K. Kwok, U. Welp, H. Minami, K. Kadowaki, and T. Yamamoto, Appl. Phys. Lett. 103, 022602 (2013).

${ }^{18}$ S. Sekimoto, C. Watanabe, H. Minami, T. Yamamoto, T. Kashiwagi, R. A. Klemm, and K. Kadowaki, Appl. Phys. Lett. 103, 182601 (2013).

${ }^{19}$ M. Tachiki, T. Koyama, and S. Takahashi, Phys. Rev. B 50, 7065 (1994).

${ }^{20}$ L. N. Bulaevskii and A. E. Koshelev, Phys. Rev. Lett. 99, 057002 (2007).

${ }^{21}$ S. Lin and X. Hu, Phys. Rev. Lett. 100, 247006 (2008).

${ }^{22}$ R. A. Klemm and K. Kadowaki, J. Phys.: Condens. Matter 22, 375701 (2010).

${ }^{23}$ B. D. Josephson, Phys. Lett. 1, 251 (1962).

${ }^{24}$ R. Kleiner, F. Steinmeyer, G. Kunkel, and P. Müller, Phys. Rev. Lett. 68, 2394 (1992).

${ }^{25}$ R. Kleiner and P. Müller, Phys. Rev. B 49, 1327 (1994).

${ }^{26}$ A. A. Yurgens, Supercond. Sci. Technol. 13, R85 (2000).

${ }^{27}$ The stand alone mesa means that the mesa fabricated by removing all the rest of the superconducting parts, leaving alone only the mesa on the substrate.

${ }^{28}$ M. Asada, S. Suzuki, and N. Kishimoto, Jpn. J. Appl. Phys., Part 1 47, 4375 (2008).

${ }^{29}$ S. Suzuki, A. Teranishi, K. Hinata, M. Asada, H. Sugiyama, and H. Yokoyama, Appl. Phys. Express 2, 054501 (2009).

${ }^{30}$ K. Hinata, M. Shiraishi, S. Suzuki, M. Asada, H. Sugiyama, and H. Yokyama, Appl. Phys. Express 3, 014001 (2010).

${ }^{31}$ H. Ito, F. Nakajima, T. Furuta, and T. Ishibashi, Semicond. Sci. Technol. 20, S191 (2005).

${ }^{32}$ E. Rouvalis, C. C. Renaud, D. G. Moodie, M. J. Robertson, and A. J. Seeds, Opt. Express 18, 11105 (2010).

${ }^{33}$ C. Walther, G. Scalari, J. Faist, H. Beere, and D. Ritchie, Appl. Phys. Lett. 89, 231121 (2006).

${ }^{34}$ B. S. Williams, Nat. Photonics 1, 517 (2007).

${ }^{35}$ S. Kumar, Q. Hu, and J. L. Reno, Appl. Phys. Lett. 94, 131105 (2009).

${ }^{36}$ T. Kashiwagi, K. Nakade, B. Marković, Y. Saiwai, H. Minami, T. Kitamura, C. Watanabe, K. Ishida, S. Sekimoto, K. Asanuma, T. Yasui, Y. Shibano, M. Tsujimoto, T. Yamamoto, J. Mirković, and K. Kadowaki, Appl. Phys. Lett. 104, 022601 (2014).

${ }^{37}$ A. C. Kak and M. Slaney, Principles of Computerized Tomographic Imaging (IEEE Press, 1988). 